\title{
Perceptions africaines du Brésil: le spectre du passé
}

\author{
Percepções africanas do Brasil: o espectro do passado
}

\begin{abstract}
African Perceptions of Brazil: The Ghost of the past
\end{abstract}
Adjai Paulin Oloukpona-Yinnon*

Résumé: Le dernier roman de Kangni Alem, Les enfants du Brésil, traite des aspects oubliés de la traite négrière entre l'Afrique et le Brésil. Le livre présente une sorte de recherche archéologique dans le monde atlantique, dans le but de dévoiler un savoir sur la tragédie de la traite négrière. L'auteur recourt à la métaphore des navires détruits pour créer sa propre incursion artistique à travers les souvenirs de l'esclavage. Combinant exploration scientifique et artistique, il se réfère essentiellement aux situations contemporaines au Brésil et au Togo, actualisant la représentation de la mémoire des esclaves et montrant de nouvelles façons d'écrire sur leurs impacts sur la vie quotidienne en Afrique. En analysant ce roman de Kangni Alem, j'essaie de montrer que ce n'est pas une entreprise discursive. L'auteur utilise des mots et des métaphores pour comprendre une réalité très spécifique de l'histoire et de la société togolaises. Pour déconstruire son message, il semble approprié d'utiliser une approche méthodologique pertinente telle que l'approche archéologique de Michel Foucault. Cela permet de lire le discours de Kangni Alem sur le Brésil en tant que discours sur l'Afrique.

Mots-clés: Littérature, esclavage, mémoire, afro-brésiliens, Brésil, Togo

Resumo: O último romance de Kangni Alem, As crianças do Brasil, trata dos aspectos esquecidos do tráfico de escravos entre a África e o Brasil. O livro apresenta uma espécie de pesquisa arqueológica no mundo Atlântico, com o fito de desvelar um conhecimento sobre a tragédia do tráfico de escravos. O autor recorre à metáfora de navios destruídos para forjar sua própria incursão artística pelas memórias da escravidão. Combinando a exploração científica

(c) EY Direito autoral e licença de uso: Este artigo está licenciado sob uma Licença Creative Commons. Com essa licença você pode compartilhar, adaptar, para qualquer fim, desde que atribua a autoria da obra, forneça um link para a licença, e indicar se foram feitas alterações. 
e artística, ele se refere essencialmente a situações contemporâneas no Brasil e no Togo, atualizando a representação da memória escrava e mostrando novas maneiras de escrever sobre seus impactos na vida cotidiana em África. Ao analisar esse romance de Kangni Alem, procuro mostrar que não é apenas uma empresa discursiva. O autor faz uso de palavras e metáforas para compreender uma realidade muito específica da história e da sociedade togolesas. Para desconstruir sua mensagem, parece apropriado usar uma abordagem metodológica relevante como a arqueológica de Michel Foucault. Isso permite fazer uma leitura do discurso sobre o Brasil de Kangni Alem como um discurso sobre a África.

Palavras-chave: Literatura, escravidão, memória, Afro-brasileiros, Brasil, Togo

\begin{abstract}
Absract: Kangni Alem's new novel Les Enfants du Brésil (The Children of Brazil) deals with forgotten aspects of the slave trade between Africa and Brazil. It is presented as a kind of archeological research in the Atlantic Ocean, an attempt to find out knowledge about the tragedy of slave trade, but the author uses the metaphor of wrecked ships to shape his own artistic exploration of present memories of slavery. Combining scientific and artistic exploration, he refers essentially to contemporary situations in Brazil and Togo, actualizing the depiction of slave memory and showing new ways of writing about its impacts on everyday life in Africa. I'm analyzing Kangni Alem's novel to show that it is not just a discursive enterprise. In fact, the author makes use of words and metaphors to grasp a very specific reality of Togolese history and society. To deconstruct his message it seems appropriate to use a relevant methodological approach like Michel Foucault's archeological theory. This allows to discover the author's discourse on Brazil as a discourse on Africa.
\end{abstract}

Keywords: Literature, slavery, memory, Afro-Brazilians, Brazil, Togo.

\title{
Introduction
}

Les relations entre le Brésil et 1'Afrique sont marquées par une abondante production de savoirs ${ }^{1}$ générés par la circulation des personnes et des connaissances entre ces deux espaces géographiques et historiques. Un des plus anciens auteurs dans le domaine est l'ethno-anthropologue et photographe français Pierre Verger (1902-1996). Passionné à la fois de l'Afrique et du Brésil, il a passé la plus grande partie de sa vie à sillonner le continent africain, puis le Brésil où il est mort et enterré en 1996. L'importante documentation qu'il a rassemblée sur plusieurs siècles d'échanges, constitue aujourd'hui une source inépuisable de connaissances dont s'inspire, entre autres, la fiction littéraire, à 
l'exemple du nouveau roman du Togolais Kangni Alem, Les Enfants du Brésil (2017), qui pose en pointillés, le problème des perceptions littéraires du Brésil par les Africains, et les significations qui s'y rattachent. C'est son deuxième roman sur le sujet ${ }^{2}$, après Esclaves (2009) ${ }^{3}$, mais sa démarche romanesque est tout à fait nouvelle, puisqu'elle se conçoit comme la construction méthodique d'un pont entre l'histoire et la littérature, le passé et le présent, en somme: une reconstitution symbolique du pont historique entre les deux rives de l'Atlantique.

\section{Les Enfants du Brésil: enjeux d'un récit}

«L'archéologie sous-marine est un sujet ardu, la preuve, même ma manière de romancer n'avait pas réussi à dérider le public du Banco do Brasil.» Ainsi commence le roman de Kangni Alem. La scène d'ouverture du récit ouvre les projecteurs sur un romancier afro-brésilien, Candinho Santana, né en Afrique, à «TiBrava, capitale de TiBrava». Il vient de terminer la présentation de son dernier ouvrage, dans l'agence d'une banque, Banco do Brasil, dans la ville de Rio de Janeiro. L'auditoire médusé par son exposé, n'ose pas poser de questions, et le conférencier, sans doute embarrassé lui aussi, est obligé de boucher les trous de silence en confessant que le thème de son roman est complexe, et que, plus complexe encore est son mode d'approche qui est celui d'un archéologue qui cherche à ressusciter dans les ruines et les épaves marines, les vérités du passé. Il ajoute, à l'endroit de l'auditoire toujours perplexe: « Je précise bien que j'ai rédigé ce récit à partir du rapport établi le 2 janvier 1842 par le capitaine Stella Régis, de la police maritime de Fremantle, Australie, sur déposition du témoin et unique survivant au naufrage du James Matthew, Monsieur Fortuleza Barbarossa. » Cette précision, loin de clarifier la compréhension des choses, les rend encore plus ambigües. En effet, comment une histoire fondée sur des données historiques aussi précises peut-elle se révéler si incompréhensible, si ce n'est pas, justement, à cause du mode de narration choisi par l'auteur qui a décidé de fabriquer de la fiction littéraire à partir d'une réalité historique obsédante que tout le monde condamne ? Il se pose donc la question: comment rendre compte d'un passé douloureux qui n'a pas été vécu de la même manière par tous les protagonistes ? Voilà toute la complexité de cette œuvre romanesque qui remue candidement le couteau dans des plaies du passé, tout en faisant jubiler ceux qui, aujourd'hui, adorent la littérature de fiction, parce qu'ils n'ont pas connu la douleur du passé qu'évoque l'auteur.

Ce roman de Kangni Alem est, à mon avis, l'illustration parfaite de la difficulté à parler aujourd'hui du Brésil en Afrique, car le sujet soulève beaucoup d'images empilées sous forme de sédiments qui se trouvent brouillées en remontant à la surface. Le passé et le présent superposés s'entrechoquent et s'embrouillent. Il m'apparaît évident que l'essai théorique de Michel Foucault, 
L'archéologie du savoir (1969), s'offre comme une clé de lecture de ce roman. La méthode archéologique de Foucault consiste à révéler au grand jour des couches sédimentaires d'un savoir, à le déchiffrer pour y déceler une pluralité de niveaux, à interroger ses discours pour y découvrir les mouvements secrets de la pensée ${ }^{4}$.

Appliquée au roman de Kangni Alem, la théorie de Foucault permet de révéler que le Brésil d'aujourd'hui a du mal à être perçu par les Africains comme il est en réalité, à savoir, un pays moderne, émergent, une puissance montante dans l'économie mondiale et la politique internationale, un membre du club dénommé BRICS (Brazil, Russia, India, China, South Africa). Vu d'Afrique, le Brésil en pleine ascension économique sur le plan mondial, reste encore figé dans l'image obsessionnelle de la traite négrière ${ }^{5}$. C'est d'abord l'histoire qui s'en est occupé abondamment, puis la littérature de fiction s'en empare désormais. Le choix du mode fictionnel adopté par l'auteur n'est ni anodin, ni fortuit: il permet toutes les libertés envers la vérité historique, tout en autorisant le silence, lorsque la vérité ne tolère pas de maquillage esthétisant. Il est donc intéressant de voir dans ce roman ce qui est dit, comment il est dit, de même que ce qui est passé sous silence, et pourquoi se fait le choix du silence. L'enjeu de la présente analyse de ce roman est essentiellement de révéler les superpositions de discours et d'images, le rôle du passé dans la compréhension du présent, ainsi que les clichés figés qui minent la réalité en pleine mutation. En somme, il s'agit de sonder la pertinence et l'adéquation du mode romanesque choisi par l'auteur, au regard des objectifs que vise son roman.

\section{Littérature et archéologie}

Dès le début du roman, le propos apparaît comme un clin d'œil implicite à la science archéologique, puisque le narrateur lui-même explique que l'objet du récit se situe dans le cadre d'un projet scientifique d'exploration sousmarine de l'UNESCO dénommé «Archeos », et qui consiste à " plonger dans les pénombres des océans et côtoyer l'histoire muette des épaves, au milieu des algues, des poissons mutiques qui [...] comprennent de façon intuitive la présence sur leur territoire des habitants de la terre: là-dessous gît la vie. ${ }^{6} \gg \mathrm{La}$ métaphore des épaves, associée à celle de l'exploration sous-marine, exprime une perception à la fois précise et neutre du travail à accomplir, mais représente aussi une formulation littéraire puissante et poignante des enjeux du récit: il s'agit bien, pour le romancier, de passer d'un projet scientifique objectif (l'exploration sous-marine) à un projet littéraire personnel (la représentation des épaves de navires négriers comme métaphore du savoir qui se trouve enfoui au fond des eaux).

Le narrateur a ensuite clarifié ce projet dans sa conférence tenue à Recife, deuxième étape de sa tournée au Brésil. Il a expliqué que ce travail d'archéologie sous-marine est, « tout compte fait, une remontée en surface 
salutaire de la mémoire des peuples d'une rive à l'autre de l'Atlantique. ${ }^{7}$ » Désormais il en parle, non seulement comme d'une mission d'exploration scientifique, mais aussi comme d'un devoir qui lui procure personnellement « une fierté inexplicable ${ }^{8}$ ». Et plus il y pense, plus il en trouve la logique: lui, Candinho l'Afro-brésilien, estime qu'il est sûrement bien placé pour accomplir cette mission devenue un devoir, puisqu'on ne peut pas, raisonnablement, la confier aux ressortissants des anciennes nations esclavagistes qui sont réticentes à parler de ce sujet:

Quelle idée de vouloir demander aux esclavagistes d'écrire l'histoire à la place de l'esclave ? N'appartient-il pas à ce dernier de remonter le cours du temps, de redescendre dans les abysses de sa douloureuse mémoire pour en exhumer tous les débris poisseux, les preuves irréfutables de sa dislocation, conséquence directe de l'humiliation imposée? Et les fouilles archéo-marines, pour moi, participaient de cette logique d'exhumation de la vérité historique. ${ }^{9}$

Encore une fois, la métaphore des épaves est pertinente et significative, puisque tout y est, au sens premier comme au sens figuré: les épaves apparaissent comme les restes d'un pont de lianes qui reliait jadis l'Afrique au Brésil. Leur existence au fond des eaux prouve qu'il y a quelque chose de perdu dans les relations Afrique-Brésil, et qu'une meilleure connaissance de ces épaves pourrait contribuer à une restauration de ces relations. Le narrateur raconte donc que sa mission dans le « Projet Archeos » consiste à

répertorier les épaves des bateaux négriers, prospecter les mers du monde pour les localiser et tenter de les remonter à la surface, ce qui se révélait souvent impossible ; [...] reconstituer l'histoire violente des hommes et des femmes qui, un jour, se sont retrouvés dans les chaînes et propulsés vers une destinée dont ces embarcations étaient, à leurs yeux étonnés, les seuls témoins tangibles. ${ }^{10}$

Le narrateur se demande même si dans ces épaves, il ne pourrait pas y avoir un des siens: "Un ancêtre ? Une parente lointaine ? ${ }^{11}$ " Le projet scientifique fait donc du projet littéraire un défi personnel, un enjeu identitaire. Dans ces conditions, aborder une telle histoire sous le mode romanesque est, certes excitant et édifiant, puisqu'il s'agit alors d'inventer aujourd'hui la fiction qu'inspire ce sujet, sans oublier l'histoire réelle jadis vécue par les protagonistes. Le parti-pris de l'auteur, consciemment assumé et parfaitement légitime, semble être de proposer de la fiction à la place de la réalité, puisqu'il consiste à « lutter contre la banalité » qui, avoue-t-il, est en même temps « une 
nécessité ${ }^{12} »$. Ce travail d'exploration sous-marine est donc, certes banal, puisque c'est son métier, mais c'est également nécessaire, s'il ne veut pas, lui-même, finir comme une épave.

On comprend donc pourquoi le narrateur prend ce travail très au sérieux. « Combien d'épaves le projet a-t-il répertoriées à ce jour ? » demanda une auditrice. «Et moi de lui citer, sans consulter mes notes, des noms, des lieux, des dates, m'évertuant toujours à trouver, par-delà la froideur des statistiques, le ton qui convenait à l'importance de chaque épave..$^{13}$ » Effectivement, à Rio de Janeiro, il avait cité une dizaine d'épaves, avec toutes les précisions qui s'y rapportent. Il en sera de même à sa conférence à Recife où il en citera une autre dizaine. Et chaque fois, « la singulière destinée de [chaque] navire ${ }^{14} »$ le passionne énormément.

Comme on le voit, ce roman sert à traduire l'obsession du passé lié aux victimes anonymes et oubliées de la traite négrière, et le désir d'exorciser ce passé. Pour le narrateur lui-même, un Afro-brésilien, donc un pur produit de ce passé, ce roman ainsi que le «Projet Archeos » qui en est le prétexte, n'est rien d'autre qu'un voyage imaginaire dans son propre passé, un « devoir de mémoire » qu'il accomplit à des fins thérapeutiques personnelles, avec la précision d'un chirurgien qui dissèque son propre passé. Mais ce qui est personnel ici devient naturellement paradigmatique pour l'ensemble des Africains qui portent encore les stigmates de plusieurs siècles de traite négrière.

Comment le romancier rend-il compte de cette réalité historique?

\section{Africanité mythique du Brésil}

En Afrique, la mémoire du Brésil est plurielle et complexe. Elle comprend, d'une part, les anciens négriers d'origine européenne ou brésilienne, leurs descendants et leurs fournisseurs d'esclaves. D'autre part, il y a les Africains mêlés à la traite, comme négrier ou comme esclaves ; d'abord " déracinés » d'Afrique, déportés au Brésil, puis retournés en Afrique, ils conservent des souvenirs mitigés de leur périple brésilien ; on les appelait communément les Retornados, mais aujourd'hui, beaucoup de gens portant des patronymes afro-brésiliens en Afrique n'ont jamais connu le Brésil et n'ont aucun lien avec les Brésiliens, sauf le nom acquis par descendance. Il y a enfin les autres Africains obligés de vivre avec la descendance des anciens négriers dont eux-mêmes ou leurs descendants ont été les victimes. Evidemment, cette mémoire du Brésil englobe aussi des gens qui n'ont rien à voir - ou ne veulent rien avoir - avec ce passé. Jacques Leenhardt parle donc de «mémoire orale et privée ${ }^{15} \rrbracket$, expression qui conviendrait mieux pour cerner le souvenir spécifique de chacune de ces catégories. Mais dans la réalité actuelle, cette mémoire concerne en premier lieu ceux qui, d'un bord comme de l'autre, conservent et entretiennent le mythe de la « terre ancestrale du Brésil ${ }^{16}$ ", comme s'en souvient, dans le roman, le personnage nommé Carnelia Esperança de Pereira, 
la « grand-mère éternelle » du narrateur, « celle qui refusa de mourir même quand elle a eu cent ans ", celle qui se fait appeler Dona Beija, nom de la célèbre héroïne d'une série télévisée brésilienne du même nom. Elle proclame: « Nous ne sommes pas d'ici », et elle chante aussi:

Galope, galope mon cheval

Dans une course sans frein

Et va dire à mon Brésil

Que de lui je me souviens. ${ }^{17}$

Au narrateur, un Afro-brésilien qui n'avait jusque-là jamais connu le Brésil, ce chant inspire une envolée pathétique, à l'approche du jour de son premier voyage: "Voir le Brésil, partir sur les traces des nôtres qui y ont vécu au temps troublé des caravelles voleuses d'âmes et de corps, ma joie n'a plus connu de limites. ${ }^{18}$ » C'est donc, pour le narrateur, une sorte de voyage initiatique aux sources qu'il n'avait jusque-là jamais connues. Lui-même, autant que ceux qu'il va y découvrir, représente une « africanité mythique » du Brésil. Dans le Brésil mythique, il n'y a donc pas que les épaves, il y a aussi ceux qui les ont causées et dont les descendants, sans le savoir ou sans le vouloir, s'en vantent en revendiquant leurs origines brésiliennes, comme Carnelia Esperança de Pereira et toute sa descendance dont fait partie le narrateur.

De l'autre côté, au Brésil même, il y a les pendants du mythe: tous les clichés sur le pays et ses habitants, tous les mythes et toutes les réalités de l'africanité supposée ou réelle, par exemple le Brésil du Vodou, avec Xangó, le dieu du Tonnerre, Yemanjá, la déesse des eaux, et Yansan, déesse des vents et des tempêtes. Il y a les adeptes du Vodou comme Luis Lavandeyra, « initié au Vodou au Nigeria ${ }^{19}{ }$, qui proclame à qui veut l'entendre: « Nous les Africains, nous devons défendre nos religions ", et qui s'indigne ensuite «Pourquoi me regardez-vous avec ces yeux-là, monsieur, est-ce parce que j'ai dit que je suis africain, alors que je suis blanc $?^{20} "$

Dans ce Brésil, il y a évidemment la réalité du racisme insidieux, par exemple celui des Blancs, implicite dans le sujet de thèse en anthropologie de Dalva Dos Santos Boivin: «L'action des maladies africaines sur la constitution des peuples du Brésil », un travail académique qui se propose d'étudier les « apports de l'A frique à la civilisation du Brésil. ${ }^{21}{ }$ " Tout un programme ... de racisme scientifique! Il y a également le racisme des Noirs comme Paula dos Santos, la directrice noire de Banco do Brasil, qui semble prêcher partout la " préférence raciale », même en amour.

Dans ce Brésil mythique, on trouve aussi, bien sûr, les mythes fondateurs de l'histoire des Noirs dans ce pays, par exemple l'évènement historique le plus connu sans doute, la révolte des esclaves musulmans qui secoua la ville de Bahia en 1835 et qui est évoquée dans ce roman à travers un de ses héros, Miguel do Sacramento, dit le Malê, " nègre immense et droit comme s'il 
marchait sur des échasses. ${ }^{22} »$ Bahia, c'est évidemment la plus africaine des villes brésiliennes, « la ville des saints chrétiens et des dieux d'Afrique ${ }^{23}$ ». Elle ne pouvait donc pas être simplement évoquée de manière expéditive dans ce roman. Comme si un Africain ne peut pas aller au Brésil sans aller à Bahia, cette ville sera la troisième étape de la tournée de conférences du narrateur, et elle va aussi prêter son cadre à une longue séquence de Candomblé:

Ah, $[\ldots]$ Bahia, la ville où croire n'avait pas de sens, puisque la voilure qui la recouvre depuis l'océan est faite de l'étoffe même dont on bâtit les mystères. Et ce depuis Cabral, depuis Magellan, depuis les jésuites et les premières importations des dieux d'Afrique sur cette terre, autrefois possédée par les dieux et les esprits des innombrables tribus indiennes. ${ }^{24}$

Bahia, « la terre où les dieux d'Afrique et les saints de la religion catholique se tutoient d'égal à égal ${ }^{25}$ ", Bahia, certes la plus africaine des villes du Brésil, mais où certains «Africains » du Brésil ne savent rien - ou ne veulent rien savoir - de leurs origines africaines. ${ }^{26}$

On note aussi bien d'autres clichés sur le Brésil: la pauvreté des bidonvilles qui contraste avec la splendeur des plages idylliques, la sensualité des femmes et la beauté virile des mecs, toute l'image d'Epinal du Brésil, « un mélange de paradoxes. ${ }^{27}$ » Il n’y a pas de Brésil sans Rio de Janeiro, sans Ronaldo, la star brésilienne du foot, dont l'immense suite construite pour sa mère, attire la fine fleur de la bourgeoise locale invitée à des réceptions somptueuses. Il n'y a pas de Brésil sans la musique brésilienne, envoûtante comme aucune autre au monde, encore moins sans sa sœur jumelle, la samba, la danse qui, au Brésil, est un délire qui mène souvent aux transes. Pas de Rio sans macumba, sans carioca, sans maracuja ou sans feijoada. Tous les clichés y passent. Pas de Rio sans Copacabana, sans femmes légendairement belles et frivoles. Pas de roman sur le Brésil sans amour au Brésil, sans femme frivole et adultère, puisque « il est vrai qu'à Rio, $[. .$.$] il existe deux week-ends dans$ la semaine, celui qui commence réellement en fin de semaine, vendredi soir, et l'autre dont le top est donné jeudi, tôt vers seize heures, le long week-end dit de l'adultère. ${ }^{28} \gg$ Alors, bien évidemment, le roman des épaves de la traite se recycle lentement en une aventure amoureuse dans laquelle deux femmes se disputent les faveurs du narrateur: "Ainsi naissent dans les villes les grandes histoires d'amour » qui, comme le " libertinage sentimental », " font des relations entre les hommes et les femmes une fête perpétuelle. ${ }^{29} »$ Finalement, il y aura même trois femmes qui se disputent les faveurs du narrateur. Cela aussi est un cliché.

Et puis, dans ce Brésil, on trouve aussi tout le reste: la nostalgie jamais assouvie des Brésiliens, la terre promise, mais jamais conquise. Et surtout, vers la fin du roman, la confession du narrateur: « Au fond, qu'étais-je venu chercher 
au Brésil, sous couvert de tournées de conférences ? L'amour d'une terre d'où venaient mes ancêtres, ou tout simplement moi-même ? Il faut croire que la réponse à ma propre question m'intéressait encore si peu..$^{30} \gg$ Il ne tardera pas à savoir qu'il était venu au Brésil, à la rencontre de son destin. Alors, une histoire d'amour prend lentement le pas sur l'histoire tout court, sans que celle-ci puisse disparaître totalement. Ainsi quand le narrateur frétille de danse face à la jeune et belle Dalva Dos Santos Boivin, celle-ci ne peut s'empêcher de lui rappeler avec plaisir et bonheur: « C'est un vieux rythme brésilien, cela remonte aux temps des esclaves. » Et Candinho de répliquer: « Vraiment, on se croirait chez moi. Même la langue du chant me rappelle une langue africaine, je ne savais laquelle. ${ }^{31} \gg$ En somme, le Brésil, c'est toujours l'Afrique, et l'Afrique, c'est toujours le Brésil! C'est pourquoi, confondues dans un même mouvement de gaîté et de bonheur, les deux histoires du roman, celle des épaves de la traite et celle de l'amour entre Candinho et Dalva, vont se fondre en une merveilleuse romance: les deux tourtereaux devenus brusquement deux divinités africaines - Xangó et Yansan - vont commencer au Brésil le bonheur de l'éternité qui s'achèvera en Afrique, à TiBrava, la ville natale de Candinho: «Xangó allait rentrer avec sa Yansan à TiBrava », « ce pays caméléon où vivre au quotidien relevait de l'héroïsme. ${ }^{32} »$ Le récit se termine comme toujours dans les contes de fée: ils vécurent heureux et ils eurent beaucoup d'enfants ; mais comme la traite négrière, trame sédimentaire du récit, fut tout, sauf un conte de fée, la fin du roman ne sera pas tout à fait celle des contes de fée: le couple n'aura pas beaucoup d'enfants, du moins pour le moment: il en aura au moins un.

Que faut-il voir dans cette fiction littéraire ? Quel sédiment se cache sous le happy end du roman? La langue de bois au sujet de l'esclavage ? Le déni de l'histoire de la traite ? Une fiction pour mieux cacher la réalité historique ? Chacune de ces questions trouve des réponses dans le roman, puisque l'auteur semble lui assigner plusieurs objectifs, le premier étant la mémoire de l'esclavage sur laquelle on ne peut pas observer le silence sans tomber dans le déni.

\section{Le discours du roman sur la mémoire des esclaves}

Ce roman sur le Brésil mythique n'occulte pas le sombre chapitre de l'esclavage. C'est le « devoir de mémoire » auquel le narrateur s'est plié dès le début du récit, sous la métaphore des épaves. Il parle donc du Brésil des esclaves, mais avec pudeur et retenue, en pratiquant l'art de la pirouette littéraire. En y regardant de près, on constate qu'il en parle, certes pas abondamment, mais puissamment et densément, par exemple lorsqu'il écrit:

La vie des nègres, esclaves des Blancs au Brésil, ne fut pas un grand feu de joie. Il y en avait qui se tuaient en mangeant de la terre, se pendaient ou s'empoisonnaient avec les 
herbes et les potions des sorciers et autres quimboiseurs. La nostalgie du pays. Certains finissaient par devenir fous, idiots. Ils agonisaient lentement. Sans trouver aucun goût à la vie, se livrant à tous les excès, abusant de l'eau-de-vie, de l'opium, se masturbant. ${ }^{33}$

Parfois, l'auteur parle aussi de la vie des esclaves avec passion et excitation, par exemple lorsque Dalva, pour faire une blague, propose à Candinho de visiter un ancien dortoir d'esclaves dans une ancienne plantation. Candinho se précipite vers le bâtiment:

Tout excité, je m'approchai du bâtiment et m'agenouillai au sol, tentant de glisser ma tête et mes épaules jusqu'à la grille du premier soupirail. Qu'espérais-je découvrir au juste, je ne sais. Des silhouettes de fantômes d'esclaves ou des spectres lumineux de Nègres suppliciés, infectés par les parasites et maladies qu'ils avaient apportés d'Afrique, parasite bicho da Costa, dysenterie, maculo, pians, tumeurs gandú, déformations osseuses du nez et du maxillaire ... ? ${ }^{34}$

De même, lorsque Jorge et Júnior, ses accompagnateurs à Recife, lui proposent de visiter, de nuit, le site d'un ancien « supermarché d'esclaves », Candinho éprouve « des émotions violentes et contradictoires » qu'il ne put s'empêcher de commenter:

Lieu fragile, mais lieu de mémoire important. Pour la première fois depuis mon arrivée au Brésil, quelque chose en moi bougea de manière sensible. J'avais les larmes aux yeux, rien que d'imaginer des hommes et des femmes privés de dignité entassés là comme des bêtes. Cela m'a pris d'un coup, une tristesse de l'âme difficile à réprimer, je me suis mis à m'imaginer là, esclave parmi les esclaves, soumis à la volonté des autres, seigneurs fortunés, aventuriers sans scrupules, décidés à m'exploiter jusqu'à la mort pour leur propre projet d'enrichissement. Seul, parmi d'autres solitudes, et surtout impuissant. Un esclave, c'est quoi finalement? Une force de la nature privée de volonté, un individu capable de produire toutes les beautés du monde mais impuissant vis-à-vis de ses maîtres, un animal de somme sans parole, sans émotion. Du moins, c'est ce que l'esclavagiste en avait fait. ${ }^{35}$

Pour la première fois, " le Brésil de nos ancêtres » lui révèle « la complexité de l'héritage qu'ils nous ont laissé ici, ces êtres fiers réduits à l'état de bêtes de somme. ${ }^{36}$ » Le Brésil le ramène donc à cette Afrique où les Afro- 
brésiliens vivent encore aujourd'hui, les mirages du Brésil du passé, comme s'ils n'avaient pas connaissance des épaves et des esclaves échoués au fond des océans. Ce passé, Candinho l'avait pourtant vu de ses propres yeux dans son pays d'origine en Afrique, lorsque, tout jeune encore, il est allé pour la première fois à Porto Seguro, et que lui fut racontée l'histoire d'une cache d'esclaves, " l'histoire de Woodhouse, [...] une preuve de l'histoire violente que nos ancêtres ont subie sur la côte. [...] Ici, en plein XIXème siècle, s'est déroulée une grande partie de la traite clandestine entre le Brésil, Cuba et les souverains de la côte des esclaves..$^{37} \gg$ Mais en Afrique, ce passé douloureux n'avait pas vraiment marqué Candinho. En effet, ils ont existé, et ils existent encore aujourd'hui en Afrique, des Afro-brésiliens qui n'ont aucune conscience de la douleur de la traite négrière. Les plus conscients d'entre eux, tout en célébrant la fierté de leurs origines brésiliennes vraies ou supposées, se décrivent, dans le roman, par autodérision comme « des peaux noires masques blancs, des héritiers de Noirs aliénés par un long séjour au Brésil, et qui se considéraient fondamentalement différents des autres Noirs par le raffinement de leur culture métisse [...], des porteurs du flambeau de la civilisation ${ }^{38} »$, ou comme les « glorieux héritiers des anciens esclaves. ${ }^{39} »$. Cette autodérision n'est en réalité qu'un miroir narcissique dans lequel certains Afro-brésiliens d'Afrique contemplent leur propre image, pour mieux la soigner et s'en délecter, et célébrer ainsi « la terre ancestrale du Brésil, [...] ce pays mythique d'où, autrefois déportés, ils sont repartis libérés du poids de l'esclavage », ce qui, comme ils le disent, « continue de fouetter la fierté de notre communauté. ${ }^{40}$ » Ils considèrent donc

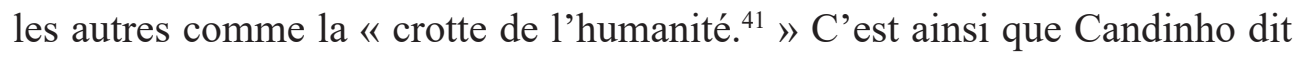
par exemple à son ami Djibril (sans savoir que ce dernier aussi descend d'un Afro-brésilien célèbre): «Je suis peut-être gros, mais je suis Brésilien. T'es qui, toi ? Petit musulman zéro, ton papa zéro, ta maman zéro. Tu sais toi ce que ça veut dire Brésilien ? ». Il récite, à l'appui de ses prétentions de supériorité, tous les noms afro-brésiliens, « des noms de gloire » qui attestent

de tout ce sang noble qui coule dans mes yeux, $[\ldots]$ cohortes
de noms qui rappellent notre douloureux passé dans le
Nouveau Monde, au temps d'un Portugal au faîte de sa
splendeur brésilienne et notre retour sur la terre des origines,
aux côtés de nos frères restés dans l'obscurité des fétiches. ${ }^{42}$

C'est le lieu de faire une digression utile sur l'autoreprésentation de l'Afro-brésilien, pour souligner quelques contradictions internes de son identité que soulève le roman.

\section{Les « Brésilien[s] de pacotille »}

Selon les propos de Candinho ci-dessus cités, l'Afro-brésilien (ou son descendant), se considère comme un «Brésilien ». Et pourtant, dans le 
roman, Velázquez, un des personnages les plus curieux, l'appelle à juste titre un «Brésilien de pacotille ${ }^{43}$ ». L'auteur construit et déconstruit ainsi le concept d'Afro-brésilien qui désigne en fait, un Africain devenu esclave de sa propre image et de son rapport avec le Brésil: ceci est valable pour l'ancien négrier (ou descendant de négrier) qui ne peut se libérer de son passé, mais vaut tout autant pour l'esclave (ou le descendant d'esclave) qui ne peut plus s'affranchir de sa conscience historique d'esclave. Quelque part dans un coin obscur de sa mémoire, chaque Afro-brésilien a conscience des horreurs perpétrées par la traite négrière à laquelle il a participé directement ou indirectement, de près ou de loin, d'un bord ou d'un autre, en tant que victime ou en tant que bourreau. Mais au lieu de se libérer de cette image qui l'emprisonne, il en a fait un vêtement de prestige, un habit de noblesse qu'il endosse avec fierté, oubliant qu'aux yeux des «Autres » (c'est-à-dire tous ceux qui ne sont pas Afro-brésiliens), cet habit représente justement le symbole des horreurs de la traite et la preuve de la responsabilité historique directe de tous ceux qui y ont participé. Chaque Afro-brésilien est probablement conscient de cette réalité ambigüe, mais s'en accommode au prix de mille contorsions. C'est pourquoi, dans le passage cité ci-dessus, Candinho récite les versets du credo afro-brésilien hérité de ses parents et grands-parents, et traite arrogamment Djibril de « petit musulman zéro », ignorant que le père de ce dernier est aussi un Afro-brésilien d'ailleurs plus illustre que son propre père à lui.

Cette fierté teintée d'ostracisme envers les autres Noirs perçus comme " la crotte de l'humanité », est soigneusement entretenue de génération en génération, sans que soient questionnés sa légitimité et son fondement. A ce sujet, le roman de Kangni Alem est d'une fécondité étonnante et insoupçonnable, car l'auteur - à travers le narrateur - réussit à confondre l'Afro-brésilien en le mettant face au sens réel de sa curieuse image. Il le confronte avec l'absurdité de sa ridicule fierté. Comme on le sait, certains Afro-brésiliens ont été des esclaves, comme Miguel do Sacramento, le père de Djibril, dont l'histoire glorieuse est abondamment racontée dans le roman. A cause de son « séjour infâmant dans le Nouveau Monde ", Miguel do Sacramento est revenu pour « revendiquer à rebours ses origines africaines..$^{44} »$ L'identité afro-brésilienne est donc une identité de substitution qui sert à voiler et occulter un passé que l'on a du mal à assumer. Si l'on exclut la richesse présumée des Afro-brésiliens, signe extérieur qui leur sert de faire-valoir, rien ne justifie leur fierté ostentatoire, bien au contraire: tout la condamne. Le personnage de Velázquez en est la parfaite illustration: il appartient - de naissance, et donc de droit - à cette communauté, mais il en a été exclu du fait des circonstances de sa naissance: fils naturel de Carnelia Esperança de Pereira, Velázquez a dû vivre caché et pauvre toute sa vie, uniquement pour que l'honneur de la famille de sa mère soit sauvé. Ceci l'a contraint à devenir un saltimbanque qui mendie son pain quotidien, au vu et au su de sa mère qui, de toute évidence, n'en a jamais éprouvé du chagrin, 
encore moins du remords. La fierté et l'honneur de la famille ont donc été mis bien au-dessus du lien de sang entre la mère et son fils. Le narrateur résume cette incongruité morale et sociale en une phrase qui sonne comme un verdict cinglant: «Oui, l'enfant n'était pas mort, et l'honneur de sa famille et celui de son amant étaient saufs. ${ }^{45}$ » Cet amant, géniteur de Velázquez, est un homme d'église devenu un prélat haut placé qui, lui aussi, a mis la morale et la religion au placard, pour mieux vivre dans le secret, sa vie de débauche, une vie de père indigne et de religieux moralement compromis, et donc condamnable. Il a fallu que Velázquez lui-même use de stratagème en organisant un spectacle de saltimbanques au cours duquel il étale au public, par allusion et dérision, l'horrible histoire de sa vie. C'est alors et seulement alors, que sa mère décide de lever le voile sur ce secret de famille jalousement gardé. Hélas, quand ce sera fait, ce sera déjà trop tard pour Velázquez qui va mourir peu de temps après cette révélation. A TiBrava, le silence coupable de Carnelia Esperança de Pereira, la plus fière des Afro-brésiliens, est un exemple de l'inhumanité dont cette communauté est capable pour préserver son honneur. Et ce n'est là qu'un des aspects problématiques de cette identité des Afro-Brésiliens. Il va de soi que leur fierté construite sur les épaves de la traite négrière, rend difficile le dialogue entre les descendants des négriers et les descendants des esclaves ${ }^{46}$. La fierté et le mensonge dans lesquels s'enferme cette communauté afro-brésilienne exacerbent le ressentiment des victimes (ou de ceux qui prétendent l'être). Face à cette situation, les exclus de tous bords ne se privent pas, s'ils en ont l'occasion, de proclamer tout haut, ce que d'autres pensent tout bas: « Ce pays appartient à tous [...]. Nous sommes un héritage de douleurs subies et partagées, un assemblage de mensonges savamment orchestrés, des mensonges que personne, jusqu'ici, n'a eu l'audace de mettre à jour. ${ }^{47} \gg$ Voilà ce que le romancier dénonce avec courage. Mais, malgré cette dénonciation, chacun se bouche les oreilles pour ne pas entendre les cris des naufragés qui n'ont pas trouvé le repos dans les épaves au fond des mers. Il y a quelque chose d'arbitraire et de révoltant dans la construction de l'identité afro-brésilienne, une sorte de déni qui falsifie l'histoire. Et c'est bien cela que montre habilement le roman de Kangni Alem qui a choisi de superposer des images du passé et du présent, l'une servant de sédiment à l'autre, l'une minant l'autre, pour mieux la dynamiter. L'auteur revisite donc à sa façon l'histoire de la rencontre du Brésil avec l'Afrique, en racontant sur le mode métaphorique ce passé lugubre figé dans l'image des épaves de morts qui hantent la mémoire des vivants. Africains et Brésiliens continuent d'aller et venir sur le pont de lianes jadis tissé au-dessus de l'Océan Atlantique, par-dessus les épaves enfouies au fonds des eaux. Ils font semblant de ne pas entendre les cris des morts venant du fond des océans Et justement, parce qu'ils font comme si de rien n'était, les morts hantent leurs voyages et leurs souvenirs, comme des fantômes tourmentés qui refusent aux vivants le droit de vivre en paix. 
Mais dans sa démarche romanesque, Kangni Alem transforme la complainte des épaves en chant d'amour. Il transforme le roman des douleurs en roman d'amour. La logique de l'auteur, c'est donc de reconnaître qu'on ne peut pas se taire sur la traite et ses conséquences, il faut donc en parler. Mais s'il faut en parler, il ne faut surtout pas accuser, car accuser l'autre, c'est déjà s'accuser soi-même. Alors, le romancier invente un mode de récit qui parle de tout, sans indexer personne. En superposant les images du présent et du passé, en présentant le passé à travers un projet de recherche scientifique à la recherche d'épaves sédimentées, l'auteur réussit le tour de force de dépassionner le débat sur la traite. Il raconte une vérité qui reste vraie, tout en étant inventée et réinventée. Selon le critique et théoricien Roland Barthes, ceci est permis par la liberté poétique, car « il n'y a rien d'étonnant à ce qu'un pays reprenne [...] périodiquement les objets de son passé et les décrive de nouveau pour savoir ce qu'il peut en faire. ${ }^{48}$ "

Mieux encore, l'auteur parvient à faire de son « roman sur le Brésil », un « roman sur l'Afrique », en opérant tout simplement une translation des représentations esquissées. L'africanité mythique du Brésil devient alors une plainte, voire une complainte sur le Togo. Sous la plume de Kangni Alem renait le Togo de la dictature quotidienne, l'Afrique toujours en pleine bataille de libération.

\section{Les Enfants du Brésil : une complainte du silence sur le présent}

Le critique littéraire togolais Guy Kokou Missodey a résumé en ces termes le roman de Kangni Alem:

Les Enfants du Brésil est une invitation aux voyages: voyage dans les fonds abyssaux, symbole de notre mémoire collective pour faire resurgir les douloureux souvenirs réprimés ou refoulés ; voyage dans les profondeurs du Brésil moderne pour révéler quelques unes de ses contradictions; et enfin, voyage dans l'univers intime de l'écrivain, son « espace du dedans », selon l'expression de Henri Michaux, pour partager avec lui ses fantasmes, entre rêve et réalité 49 .

La double expression " l'univers intime de l'écrivain, son 'espace du dedans' » se révèle ici très judicieuse et mérite d'être sondée. En lisant attentivement le dernier chapitre du roman, on constate que son titre, « Jours heureux à Tibrava ", est conçu pour signifier exactement le contraire de ce qu'il dit. En effet, les chapitres précédents annonçaient déjà des « hostilités », puisque: « quinze hommes en treillis, brandissant des matraques, avaient sauté d'un camion militaire et avaient foncé sur la foule ${ }^{50}$ " (scène de bastonnade récurrente au Togo depuis plusieurs années). Ce dernier chapitre se termine d'ailleurs par une phrase emblématique de l'histoire du Togo: « La nuit est 
longue, mais le jour vient ${ }^{51} »$, parole symbolique de la libération du Togo du joug colonial en 1960. La complicité du lecteur est requise ici pour comprendre cet appel du pied à l'histoire. En effet, il est logique de penser que le titre du dernier chapitre («Jours heureux à TiBrava ») se réfère explicitement au happy end du roman symbolisé par le couple heureux Candinho et Dalva, retourné en Afrique, en terre natale du mari. Mais, en réalité, il y a aussi d'autres références, ainsi que des allusions implicites à d'autres sujets, par exemple, quand le narrateur dit en traversant les rues de la capitale: « Je me rendis soudain compte que cette cité n'était plus la même depuis la mort de son plus illustre fils ${ }^{52}$ ", c'est une allusion directe à la personne qui a prononcé la phrase symbolique précédemment citée. Il en est de même pour une autre allusion: "Si cette ville [TiBrava] pouvait parler, me dis-je, que raconterait-elle aujourd'hui au plus énigmatique de ses citoyens, disparu trop tôt $?^{53} »$ Cette autre expression ( « au plus énigmatique de ses citoyens, disparu trop tôt ») se réfère également à la même personnalité historique de la précédente citation ( « son plus illustre fils »). Dans ce dernier chapitre du roman, tout le récit semble donc tourner autour d'un personnage - ou plus exactement : d'une personne - qui reste aussi énigmatique qu'emblématique. Un coin du rideau est levé dans l'épilogue du roman, construit de bout en bout sur un ton lyrique, poétique et politique. C'est de la prose lyrique sur le Togo au présent. Le lecteur avisé reconnaît facilement dans ce tableau codé, des lieux et des personnages du Togo et de sa capitale Lomé ; par exemple lorsque le narrateur dit: «Lieu de mémoire, mon cimetière marin. Ci-gît B.B., la chanteuse mythique morte à l'âge où l'on commence la vie [...], je ne t'oublierai jamais ${ }^{54} »$, nul doute qu'il s'agit d'un clin d'œil, à la mémoire de la chanteuse togolaise Bella Bellow. Mais le récit se poursuit:

Résistance souterraine, rejet non-dit de toute mémoire non scellée dans la matière dont on fait l'assentiment populaire, comme les horribles stations dont ils ont parsemé mes carrefours: la statue toc en bronze d'un commandeur minègre mi-coréen, le square Fréau Jardin avec ses fleurs desséchées et son kiosque à musique colonial transformé en poste de guet pour les militaires du nègre en bronze, la Colombe de la Paix, symbole erroné de nos tranquillités enterrées, voici des saisons, et remplacées par le bruit, la fureur. Leurs lieux de mémoire ? Tâches grises sur l'imaginaire de mes entrailles.

Tokoin

Promontoire idéal pour découvrir mes entrailles. Comment peut-il en être autrement ? Comme ils disent, mes enfants, nous vivons dans la plus petite ville du monde, gros village cerclé d'eau où tout le monde sait qui cocufie qui et que même l'utérus d'une nonne à demi-vierge changera peu le cours de nos violences. Ah, cette ville et la violence $!^{55}$ 
Cette tirade pleine d'allusions, d'indices et de repères, renvoie implicitement à la ville de Lomé qui n'est pas explicitement nommée, mais est parfaitement reconnaissable dans ses oripeaux, puisque, au milieu de la tirade, est planté comme un fanal, le quartier emblématique de Tokoin, comme la Cathédrale Notre-Dame au cœur de Paris, la Tour de Big Ben au centre de Londres, la Statue de la Liberté à New York ou la gigantesque statue du Christ Rédempteur dans la Baie de Rio. En même temps, la tirade résonne comme un réquisitoire contre la dictature qui a fait de cette ville une « cité-martyre », et du pays entier une nation-otage. Le tout est couronné par l'exclamation en guise d'indignation : «Ah, cette ville et la violence !»

Qui peut nier, après tant de précisions, qu'il s'agit ici d'une dénonciation de la violence imposée aux habitants de cette cité ? En somme, l'épilogue décrit tout, sauf des « Jours heureux à Ti-Brava ». Il s'agit plutôt d'une complainte lugubre sur le présent devenu plus sombre que l'histoire des esclaves au Brésil. Ici comme dans le reste du roman, il y a donc des sédiments de discours séditieux qu'il faut aller repérer au fond du discours du narrateur, ainsi que dans les recoins de l'histoire du Togo. Le pont reliant le « roman sur le Brésil » et le « roman sur le Togo » se trouve ainsi explicitement établi. Le passé rejoint le présent. Le Brésil rejoint l'Afrique, là où l'avait laissé le pont de lianes cassé. Le narrateur, Afro-brésilien en voyage initiatique aux sources de son identité problématique, retourne à la triste réalité de son vrai pays.

En extrapolant, sans exagérer, on pourrait dire que la traite négrière qui, dans le roman de Kangni Alem, est appelée, d'une manière ironiquement et faussement consensuelle, « une histoire à la fois glorieuse et honteuse $\mathrm{e}^{56}$ », est à l'image de l'histoire du pays du narrateur, et de l'auteur aussi : elle est glorieuse au regard de ceux qui se prennent pour ce qu'ils ne sont pas, elle est honteuse, vue par ceux qui en sont les victimes. Selon le même raisonnement, on peut postuler que beaucoup de remarques du roman sur le Brésil sont valables pour le Togo. Par exemple lorsque le narrateur, dans sa conférence à Recife, dit de son travail d'archéologie sous-marine que c'est « une remontée en surface salutaire de la mémoire des peuples d'une rive à l'autre de l'Atlantique », on devrait comprendre aussi que TiBrava (alias Togo) est sans aucun doute sur l'une de ces deux rives. Ce n'est donc pas seulement un « roman sur le Brésil », mais aussi un « roman des deux rives » de l'Atlantique, donc autant sur l'Afrique que sur le Brésil. Et lorsque le narrateur ajoute: " Arriver à pouvoir se penser à travers ce que les coques pourries des caravelles et des bricks racontent, était tout aussi vital que l'invention de mythologies justificatives au quotidien pour les descendants d'esclaves ou d'utilisateurs d'esclaves ${ }^{57}$ ", cette déclaration s'adresse aussi aux Africains, à propos du « devoir de mémoire » devenu vital pour tous.

Mais l'épilogue du récit montre aussi qu'il ne se réfère pas seulement au passé, mais que le roman est conçu avec un «mode d'emploi » spécifique 
qui est politique. Pour des raisons de stratégie narrative ou d'actualité politique, l'objet principal du dernier chapitre du roman n'est pas clair et son personnage central - ci-dessus évoqué - n'est pas, et ne peut pas être nommé. Mais grâce aux allusions bien calibrées, tout lecteur avisé sait qu'il s'agit du Togo et de « son plus illustre fils »), le «plus énigmatique de ses citoyens, disparu trop tôt» ) - le premier Président du Togo, Sylvanus Olympio qui reste incontestablement le plus illustre des Afro-brésiliens du Togo, le plus emblématique des « Enfants du Brésil » au Togo, celui qui incarne le mieux ce que le narrateur appelle « la fierté de notre communautés8 », celui qui, plus d'un demi-siècle après son assassinat en 1963, continue d'être aujourd'hui, une 'épave vivante' dans l'histoire de ce pays, puisque sa dépouille repose encore en terre étrangère, au Bénin, et attend d'être rapatriée au Togo, afin que les morts de la lutte pour l'indépendance reposent en paix et que leurs descendants vivants puissent faire le deuil du passé. Une telle lecture politique du roman de Kangni Alem me paraît parfaitement justifiée, au regard du sujet qui est très délicat (la traite et ses traces persistantes, la dictature et ses forces omniprésentes), et surtout au regard du mode original choisi pour en parler : la métaphore des épaves. Au cœur du roman se trouve donc la présence obsédante du présent autant que celle du passé. À chacun donc d'y lire le temps qu'il désire.

Enfin, en guise de conclusion, on pourrait bien voir aussi dans le roman de Kangni Alem une réponse de la littérature togolaise à la déclaration de 2010 sur la nouvelle politique brésilienne en Afrique.

\section{Conclusion: Les relations Afrique-Brésil aujourd'hui}

Le Brésil, puissance montante dans l'économie mondiale, membre du Club très courtisé des " pays émergents ", souhaite devenir pleinement un partenaire privilégié de l'Afrique, pas seulement dans le domaine de la culture, où les échanges sont fréquents et réguliers ${ }^{59}$, mais dans tous les domaines possibles. Au cours d'un périple en Afrique en 2010, le Président brésilien d'alors, Luiz Inácio Lula da Silva, a déclaré en juillet, au Cap Vert: « Le Brésil n'a pas les moyens de payer sa dette à l'Afrique qui lui a donné sa culture, sa façon d'être, une de ses couleurs. A Gorée, j'ai demandé pardon à l'Afrique. [...] L'Afrique est une priorité pour le Brésil ${ }^{60}{ }_{\eta}$. Lula a effectivement mis en œuvre avec presque tous les pays africains une nouvelle politique de coopération que son successeur, Dilma Rousseff, a poursuivie. Entre l'Afrique et le Brésil, il existe donc désormais un nouveau pont moderne au service de la « coopération sud-sud ». Cela suppose que l'ancien « pont de lianes » créé par la traite négrière, et qui a engendré tant d'épaves, soit symboliquement rompu, au profit du nouveau pont. Il me semble que le discours de Lula en 2010 constitue cette rupture symbolique. Mais force est de constater que, malgré tous les efforts politiques actuels, malgré les nombreux projets économiques et les passerelles culturelles vivantes entre l'Afrique et le Brésil, le souvenir - conscient ou 
inconscient - lié à la traite, obstrue toujours la voie de la coopération et rend quasi impossible une réconciliation du passé avec le présent: le passé refuse d'être passé. Puisque le roman de Kangni Alem sur le Brésil devient en fin de compte un roman sur l'Afrique, il me semble que l'auteur suggère subtilement aux Africains de regarder leur histoire en face, et de l'assumer sans concession. C'est seulement à ce prix qu'il sera possible de gagner la bataille du présent face au spectre de l'histoire et aux fantômes du passé. Cela veut dire qu'il est urgent de dépasser les perceptions réductrices du Brésil, pour le regarder comme un pays 'normal', qui a une histoire certes douloureuse, mais n'est pas seulement peuplé de Noirs. Comme l'a fait remarquer le Président Lula dans son discours de 2010, l'Afrique a donné au Brésil « une de ses couleurs »,mais une seule parmi tant d'autres. Le Brésil est un immense territoire habité par des peuples et des cultures de toutes les origines et de toutes les couleurs, une puissance du futur, pleine d'espoir, donc avec laquelle on peut coopérer sans réserve.

A quand donc une relation décomplexée de l'Afrique avec le Brésil?

\section{Bibliographie}

ALEM, Kangni: Les Enfants du Brésil. Lomé/Abidjan: Les Editions Graines de Pensées/ Frat'Mat Éditions, 2017.

CHAMOISEAU, Patrick: L'esclave vieil homme et le molosse. Roman. Paris: Gallimard, 1997. DORIGNY, Marcel: Les abolitions de l'esclavage. Paris: Presses Universitaires Vincennes, 1998.

FOUCAULT, Michel: L'archéologie du savoir. Paris: Gallimard, 1969.

LEENHARDT, Jacques: Introduction à la pluralité interprétative des récits. In: M.-T. MÄDER et al. (ed.): Brücken bauen. Kulturwissenschaft aus interkultureller und multidisziplinärer Perspektive. Bielefeld: Transcript, 2016, pp. 419-426.

M'BOKOLO, Elikia: L'Afrique entre l'Europe et l'Amérique: le rôle de l'Afrique dans la rencontre de deux mondes (1492-1992). Paris: Éditions Unesco, 1995.

MONGO-MBOUSSA, Boniface: Les malentendus de la perception littéraire. Africultures $n^{\circ}$ 1/octobre 1997, pp. 24-27, 1997.

RICOEUR, Paul: Le Conflit des Interprétations. Paris: Seuil, 1969.

VERGÈS, Françoise (dir.): Exposer l'esclavage: méthodologies et pratiques. Actes du Colloque international en hommage à Edouard GLISSANT (1928-2011), 11,12 13 mai 2011 au musée du quai Branly. Africultures n 91: Dossier, 2013.

\section{Notes}

* Université de Lomé. Lomé, Togo. E-mail: pyinnon@gmail.com

1 La présente contribution concerne un volet du projet de recherche sur « Échanges et transmission des savoirs entre l'Afrique et le Brésil », au sein du Labo-ARTELI à l'Université de 
Lomé, Togo. Ce projet porte sur les diverses problématiques des relations afro-brésiliennes historiques et contemporaines

2 Kangni Alem est auteur d'une quinzaine d'œuvres littéraires dont des pièces de théâtre, des romans, nouvelles et essais

3 Dans Les Enfants du Brésil on retrouve plusieurs personnages du roman Esclaves, et certains passages de celui-ci servent d'intertextes à celui-là

4 L'archéologie du savoir forme avec Histoire de la folie (1962) et Les Mots et les Choses (1966), la triade d'essais de Foucault sur le questionnement des méthodes d'acquisition et de transmission du savoir en sciences humaines et littéraires. Grâce à Foucault et à son «archéologie des sciences humaines», il est possible d'analyser pratiquement n'importe quel système de pensée, à travers les textes qu'il a générés. Sa théorie permet en occurrence de retrouver dans les documents anciens ou contemporains sur la traite négrière, les « traces tombées hors du temps et figées maintenant dans leur mutisme ». (cf. Bernard Valade: www.universalis.fr/ encyclopedie/l-archeologie-du-savoir (consulté le 17 octobre 2017)

5 Haïti, dont l'histoire est également très liée à la traite négrière, souffre aussi de la même perception négative de son image, mais l'instabilité récurrente dans ce pays, les révoltes persistantes et les fréquentes catastrophes naturelles qu'il a connues, confirment et justifient ce cliché négatif, ce qui n'est pas le cas du Brésil.

6 Alem 2017, p.9

7 Ibidem p. 105

8 Ibidem p.108

9 Idem

10 Ibidem p.10

11 Idem

12 Idem

13 Ibidem p.14

14 Ibidem p.15

15 Leenhardt 2016, p.419

16 Alem 2017, p.12

17 Ibidem, p.13

18 Idem

19 Ibidem, p.14

20 Ibidem, p.17

21 Ibidem, p.52

22 Ibidem, p.46

23 Ibidem, p.138

24 Ibidem, p.135

25 Ibidem, p. 121

26 Ibidem, p. 123 
27 Ibidem, p.23

28 Ibidem, p. 25

29 Ibidem, p. 26

30 Ibidem, p. 144

31 Ibidem, p. 28

32 Ibidem, p. 152

33 Ibidem, p.72

34 Ibidem, p.74

35 Ibidem, p.111f

36 Ibidem, p. 113

37 Ibidem, p.66f

38 Ibidem, p.31

39 Ibidem, p.37

40 Ibidem, p.12

41 Ibidem, p. 38

42 Ibidem, p. 37

43 Ibidem, p. 47

44 Idem

45 Ibidem, p. 181

46 C'est le sujet d'un récent documentaire «L'esclavage, et après ... » de Didier Guedou, lauréat du Grand Claude Vernon et Gislaine Dupont, édition 2016

47 Alem 2017, p.157

48 Barthes 1966, p.9

49 Sur www.togocultures.com/les-enfants-du-bresil-un-delice-a-consommer-sans-moderation/ (consulté le 23.10.2017)

50 Alem 2017, p.175

51 Ibidem, p.184. C'est la formule historique prononcée au soir du 26 avril 1960 par le premier Président du Togo, Sylvanus Olympio (un Afro-brésilien), au moment d'allumer la flamme symbolique et de proclamer solennellement l'indépendance du Togo dont il sera le premier président de 1960 à 1963

52 Alem 2017, p.191

53 Idem

54 Ibidem, p.194

55 Idem

56 Ibidem, p. 118

57 Ibidem, p.105 


\section{Ibidem, p.12}

59 Des échanges d'artistes entre le Brésil et l'Afrique sont fréquents et réguliers. Les séries télévisées brésiliennes à grand succès, les fameux telenovelas, se déversent chaque année sur le marché africain

60 Propos du Président Lula au Cap-Vert en juillet 2010, lors d'un grand périple africain, cités in: Houlaïfat Said Omar: "Le Brésil: place d'une puissance émergente en Afrique ». In: Note de recherche, Politiques, stratégies \& diplomaties économiques, 30 janvier 2015, sur www.thinkingafrica.org/V2/team (consulté le 23.10.2017).

Recebido em: 20/12/2017

Aprovado em: 07/02/2018 